\title{
Guidelines for the Management of Acne Vulgaris
}

\section{W Sinclair}

Head: Department of Dermatology, University of the Free State, Bloemfontein

Corresponding author, email: absSinclairW@ufs.ac.za

\section{Abstract}

Background: Acne vulgaris is a very common skin condition that, in the vast majority, can and should be effectively managed by general medical practitioners. Several guideline documents for management thereof exist and there is an overwhelmingly large, confusing literature bank available on the topic.

Methods: This article summarises and simplifies the main guideline documents that have been published over the last few years to present the results in an algorithmic approach to the treatment of the different types and grades of acne.

Disclaimer: Adherence to these guidelines will not ensure successful treatment in every situation and should not be deemed inclusive of all proper methods of care or exclusive of other methods of care reasonably directed to obtaining the same results.

\section{Introduction}

Acne vulgaris is the most common skin ailment in humans. It is a chronic disease with potentially severe impact on the quality of life of young people. Most cases are of mild severity and can and should be managed by the general practitioner (GP). It is therefore essential that GPs are well versed in the pathogenesis, clinical diagnosis, grading of severity, treatment algorithm and pharmacology of the medications used for acne.

The guidelines presented below are based on the latest documents generated by the Global Alliance for the Improvement of Outcomes in Acne Treatment $2007^{1}$ and $2009^{2}$, as well as the European Academy of Dermatology in $2012^{3}$ and $2016 .{ }^{4}$

\section{Pathogenesis of acne vulgaris}

Basic knowledge of the pathogenesis of acne is essential for practitioners, as treatment decisions have to be based on medication that will affect as many as possible of the factors involved. The more different pathogenetic factors are simultaneously addressed, the better the outcomes. On the other hand, two different drugs that address the same factors will not have an additive effect.

The below table outlines the relevant pathogenetic factors and the medications that will address each factor.

There are numerous drugs that may worsen acne, mostly hormonal preparations, and these should be discontinued as part of management. The most important ones are:

Table 1. Main pathogenetic factors in acne and corresponding treatments

\begin{tabular}{|c|c|}
\hline Pathogenetic Factor & Relevant Medication \\
\hline Androgen stimulation of sebaceous glands & Cyproterone acetate; Drosperinone \\
\hline Hypersecretion of sebum & Oral Isotretinoin \\
\hline $\begin{array}{l}\text { Hyperkeratosis and occlusion of the duct that drains sebum into the hair } \\
\text { follicle }\end{array}$ & Topical retinoids \\
\hline The formation of the invisible microcomedo & Topical retinoids \\
\hline $\begin{array}{l}\text { Inflammatory mediators (Interleukin 1, etc.) released after stimulation of } \\
\text { toll-like receptor } 2 \text { by Propionibacterium acnes }\end{array}$ & Topical retinoids; oral isotretinoin \\
\hline $\begin{array}{l}\text { Neutrophilic response to rupture of comedones and inflammation } \\
\text { induced by free fatty acids in sebum }\end{array}$ & Oral cyclines; oral and topical dapsone; oral macrolides \\
\hline Proliferation of $P$. acnes & Oral cyclines; oral macrolides; topical benzoyl peroxide \\
\hline Inflammatory tissue damage by matrix metalloproteinases & Oral cyclines \\
\hline
\end{tabular}


- Progesterone containing contraceptives like intramuscular medroxyprogesterone acetate or norethisterone enanthate

- Implantable progesterone (etonogestrel) pellets

- Progesterone (levonorgestrel) releasing intra-uterine devices

- Combination oral contraceptives that contain levonorgestrel as progestogen

- Oral and topical corticosteroids

- Anabolic steroids

- Vitamin B12 (especially the high dose, intramuscular products)

- Lithium

- Diphenylhydantoin

Other factors that worsen acne are mostly related to overhydrating the skin with (unnecessary) oily moisturising creams and masks. A skin suffering from acne does not require moisturising at all, unless some other pathology is also present.

\section{Clinical diagnosis of acne vulgaris}

This is usually simple when basic rules are applied. It can present with varying numbers of open and closed comedones, inflammatory papules, pustules, nodules, cysts, ulcers and scars. The face is practically always involved, the back and chest frequently, the arms and thighs rarely.

As a rule, comedones have to be present to be sure of the diagnosis, because there are several other conditions that may mimic acne, but their eruptions should not contain comedones. The only exception is acne induced by topical corticosteroids, which may show comedones, but it is not regarded as acne vulgaris.

Conditions that feature in the differential diagnosis of acne vulgaris are:

- Acne rosacea

- Steroid induced acne

- Bacterial folliculitis

- Pseudofolliculitis barbae

- Eosinophilic folliculitis

- Demodiccidosis

- Papulonecrotic tuberculid

- Folliculotropic cutaneous T-cell lymphoma.

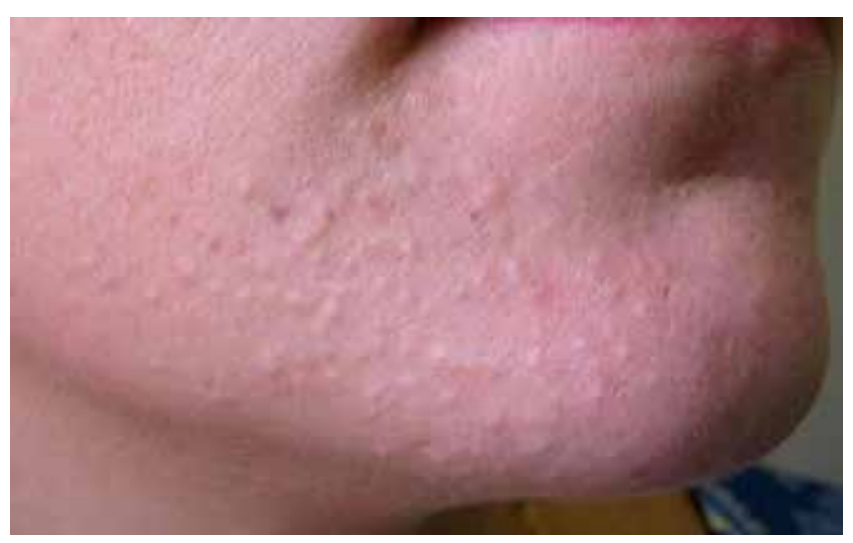

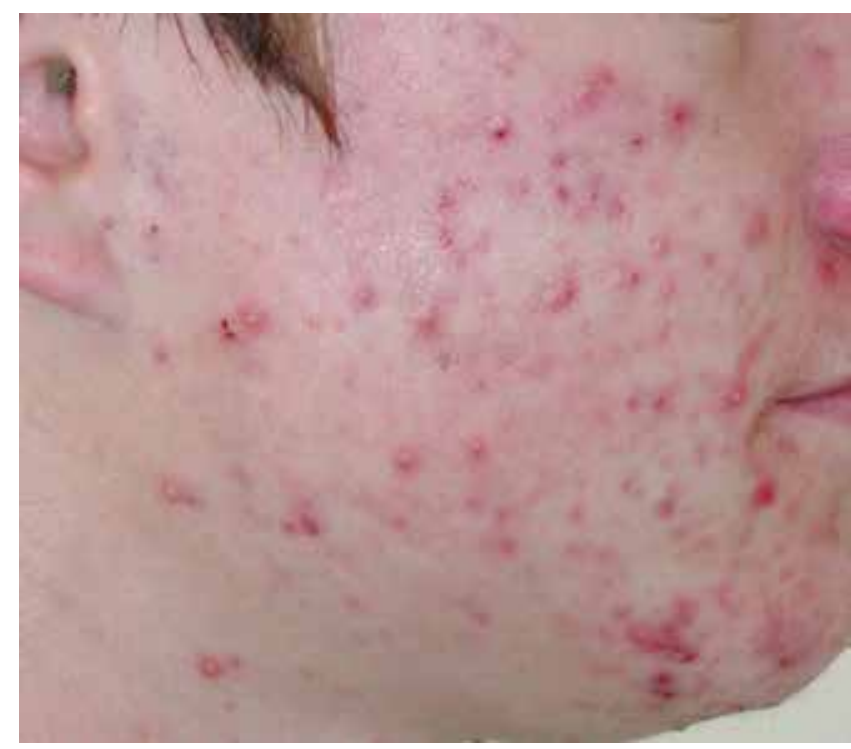

Picture 2: Inflammatory papules and small pustules

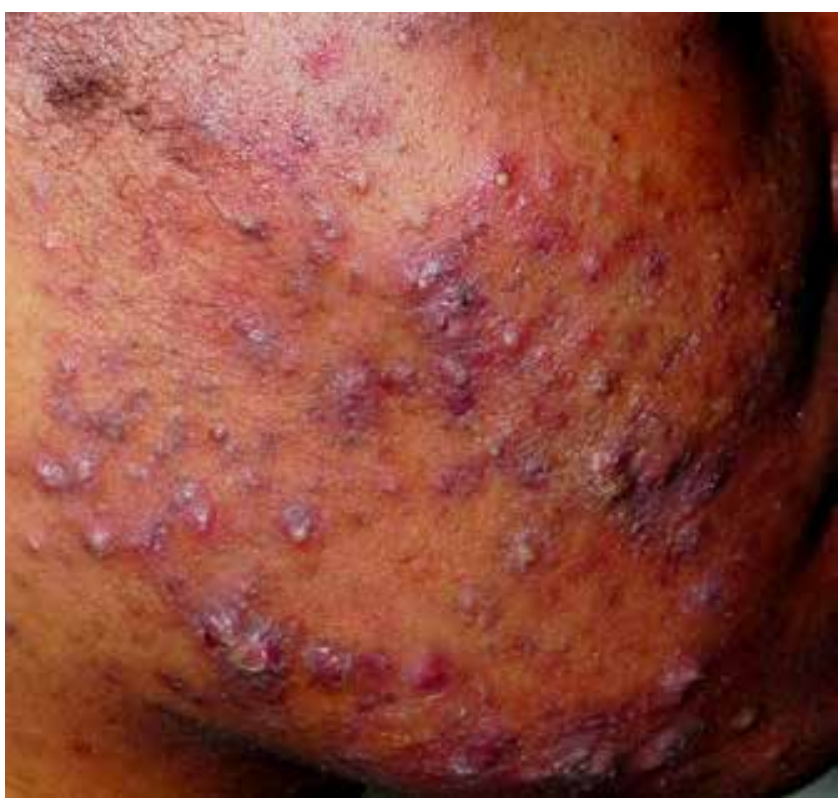

Picture 3: Pustules

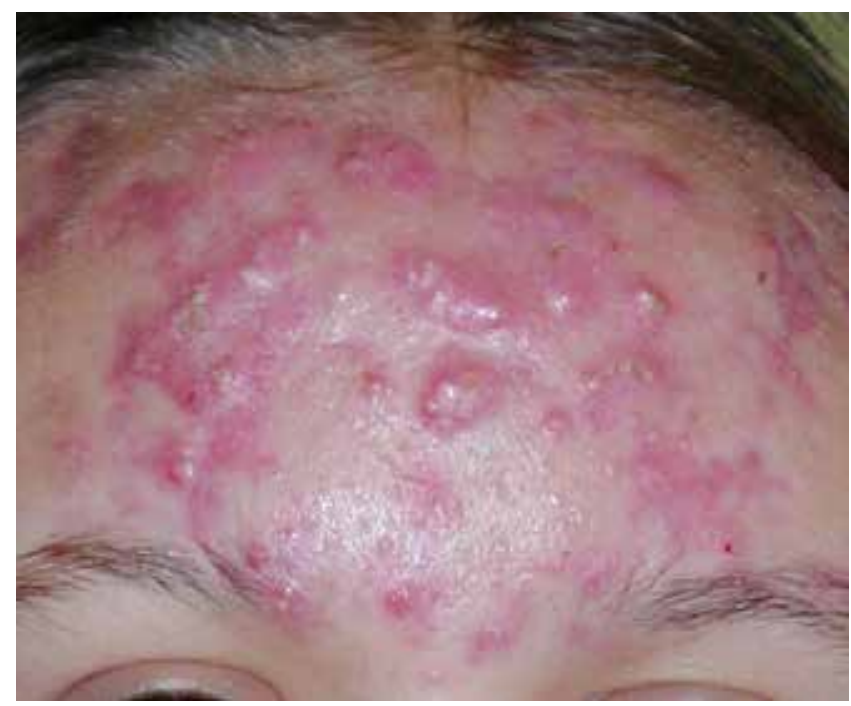

Picture 4: Nodules and cysts 


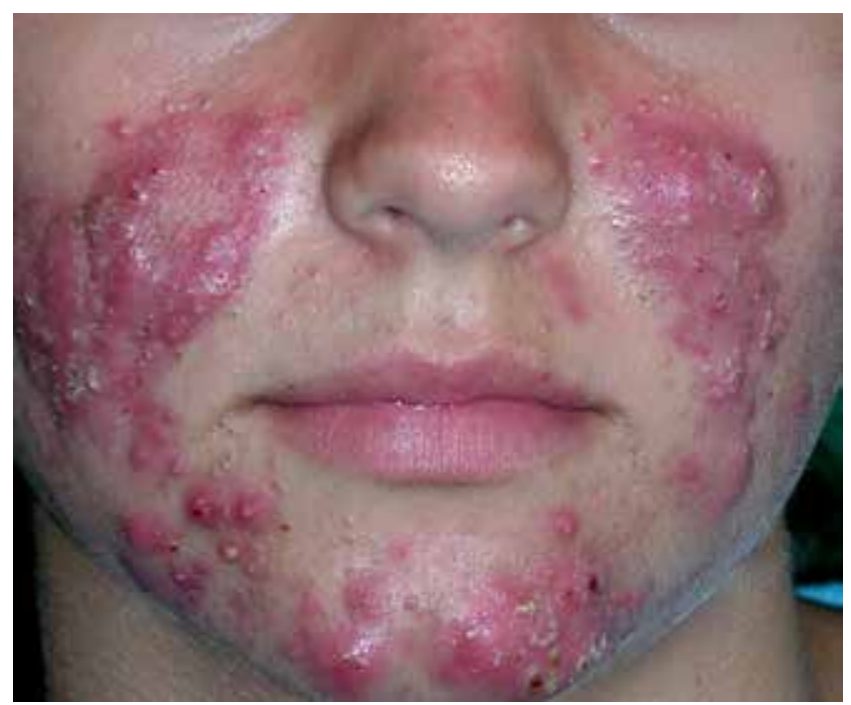

Picture 5: Conglobate acne

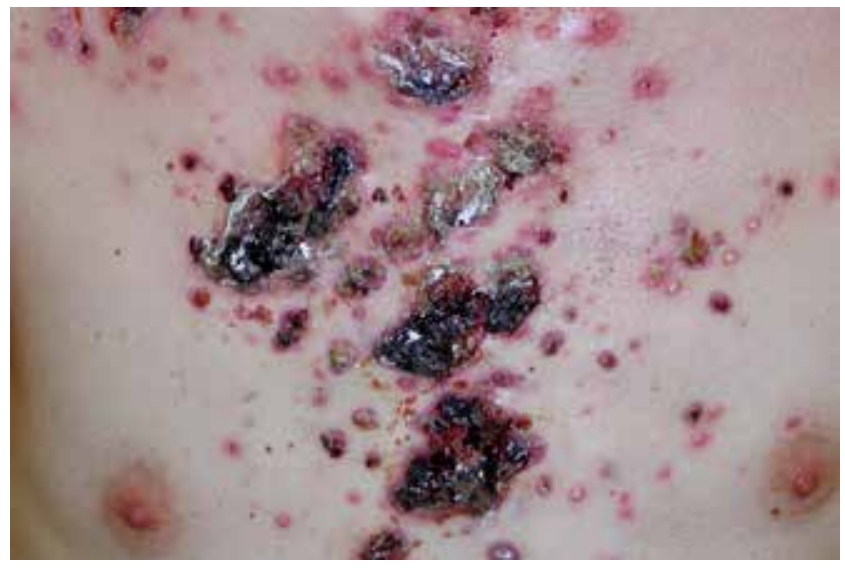

Picture 6: Ulcerating acne (Acne fulminans)

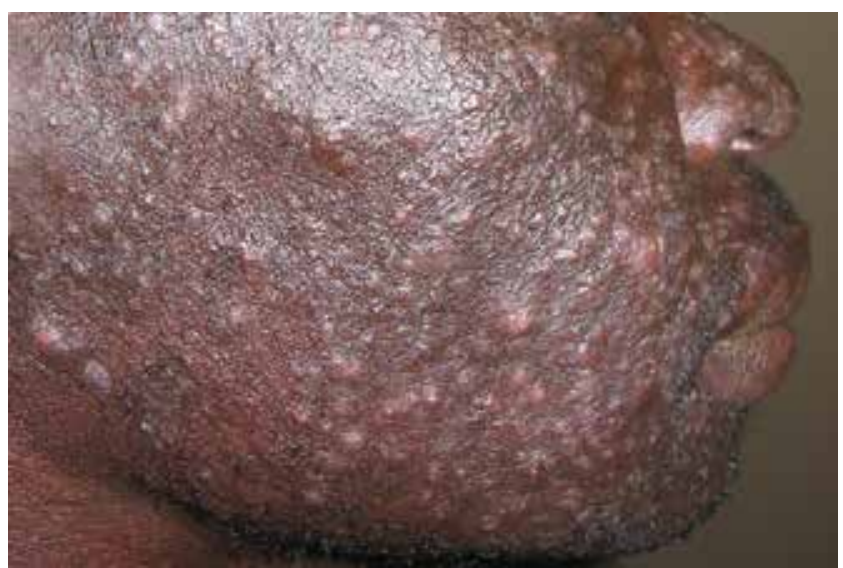

Picture 7: Folliculotropic cutaneous T-cell lymphoma resembling acne

\section{Grading of severity of acne vulgaris}

This is essential as it will be the major parameter that will determine treatment choice. Several grading systems for acne are propagated, mostly based on series of photographs on which the severity is graded rather subjectively. This does not have much practical value and should not be used for treatment decisions.

Grading should be based on the types of lesions present, not on the number of lesions. For example: If only comedones are present, it is Grade 1 acne, even if there are 10000 present; this will look very severe on a photograph, but the patient should still respond the same to topical retinoids as one with only 10 comedones and there will be no reason to use more potent or systemic treatment.

We thus propose the grading system in Table 2. It is easy to use, reproducible between observers and translates directly into treatment choices. ${ }^{5}$

\section{Treatment algorithm for acne vulgaris ${ }^{4}$}

Proposed treatment options for the different grades are as follows:

Grade1: Drug of choice: Topical retinoids (adapalene, tretinoin or isotretinoin)

Alternatives: Topical benzoyl peroxide; topical azelaic acid; comedo extraction for persistent, large comedones

These preparations should be applied daily over the whole of the affected areas and treatment should continue even after clearance. "Spot treatment" is ineffective.

Grade 2: Method of choice: Topical retinoids plus antimicrobial preparations:

- For superficial papules: Topical adapalene/benzoyl peroxide (BPO) combination product

Alternatives: Topical retinoid at night, topical benzoyl peroxide in the morning; topical retinoid at night plus clindamycin/benzoyl peroxide combination product in the morning.

Topical antibiotics should never be used alone!

- For deep papules: Topical retinoid plus oral cyclines: Lymecycline, minocycline or doxycycline

Alternatives: Topical retinoids plus oral contraceptives that contain cyproterone acetate or drosperinone (for women only).

Table 2. Grading of acne severity

\begin{tabular}{ll}
\hline Grade 1 & Comedones only \\
Grade $\mathbf{2}$ & Comedones + red papules \\
\hline Grade 3 & Comedones + red papules + pustules \\
Grade 4 & Comedones + red papules + pustules + nodules / cysts / conglobate lesions \\
\hline Acne fulminans & The above + ulceration + fever and other systemic symptoms \\
\hline
\end{tabular}

The presence of significant scarring places grading in the next higher category of severity. 
Topical retinoid plus oral macrolides for young patients or where unacceptable side-effects from cyclines were found.

Topical retinoids plus dapsone as second line

Systemic antibiotics should be used for a minimum of three months, but preferably not for longer than 4 months, to avoid the development of antibiotic resistance. During the fourth month, topical benzoyl peroxide or zinc should be added as anti-resistance agents.

There is no scientific rationale for using antibiotics (topical or systemic) as monotherapy.

Grade 3: Method of choice: Topical retinoids plus oral cyclines as mentioned above

Alternatives: Topical retinoids plus combined oral contraceptives containing cyproterone acetate for women.

Topical retinoids plus dapsone

Grade 4: Method of choice: Oral isotretinoin (OIT) as a full course (see below)

Alternatives: Topical retinoids plus combined oral contraceptives containing cyproterone acetate, with added cyproterone acetate as $10 \mathrm{mg}$ per day on days 5 - 19 of the menstrual cycle

Topical retinoids plus dapsone $100 \mathrm{mg}$ per day

\section{Maintenance treatment}

After successful clearance of acne, maintenance treatment is almost always necessary. The only exception is where a full course of oral isotretinoin may have "cured" the condition, which happens in $38 \%$ of cases treated as such. Maintenance should continue until the patient clearly never makes any new acne lesions, on average at around 25 years of age. Acne continues after the age of thirty in $14 \%$ of cases and after forty in $1 \%$ of patients. Maintenance can safely continue until then.

Topical retinoids are perfect for maintenance and are the only products registered for this indication, as they effectively eradicate the invisible inflammatory and non-inflammatory microcomedones, the origins of all acne lesions. No other medication does this at present.

OIT is not registered for the maintenance treatment of acne and such use, commonly applied, is off-label and at present not defensible. The most common reason for this inappropriate use of OIT is the neglect to use topical retinoids correctly.

Topical retinoids can be combined with topical BPO (as a single combination product) or separately if the patient continues to make superficial inflammatory papules. BPO alone is poor maintenance because it does not remove microcomedones and does not have anti-inflammatory effects like the retinoids do. Azelaic acid would be the third choice for maintenance if topical retinoids cannot be tolerated. BPO/clindamycin combination can also be used, but such long-term use of topical antibiotics is undesirable.

\section{Oral isotretinoin}

Present guidelines dictate strict indications for the use of this drug. They are the following:

- Grade 4 acne vulgaris

- Lesser grades of acne, that failed treatment with:

- Topical retinoids plus 3 months of oral cyclines, or Topical retinoids plus 4 cycles of oral contraceptives containing cyproterone acetate or drosperinone;

- Lesser grades of acne with significant scarring, to prevent further scarring; note that OIT does not remove scarring and can worsen scarring in some individuals;

- Dysmorphophobic patients who request the drug, especially when threatening self-harm;

- Gram-negative folliculitis after prolonged antibiotic use.

In Europe and the USA, oral isotretinoin may never be used as first line treatment for acne vulgaris, but in South Africa we may still use it in selected cases, as indicated above.

\section{Dosage of OIT:}

A full (potentially curative) course of this drug is administered at $0,5-1,0 \mathrm{mg} / \mathrm{kg}$ per day until a total cumulative dose of $120 \mathrm{mg}$ per $\mathrm{kg}$ is reached. This can be extended to $150 \mathrm{mg} / \mathrm{kg}$ if the acne has not yet cleared.

Cure is by no means guaranteed and one should never promise a patient a cure, because it happens in the minority of cases.

There is no official maintenance dose for OIT, as mentioned above, but the South African guidelines allow for "pulse dosing" where $20 \mathrm{mg}$ per day is used for the first 7 calendar days per month, indefinitely.

Prolonged "low dose" OIT is a form of maintenance dosing used mostly for convenience, is off-label, not supported by evidence, its safety has not been proven, has potentially serious sideeffects and in most cases, these patients could have been easily maintained safely on topical retinoid combination products.

\section{Side-effects of OIT:}

Teratogenicity. This is the best known serious adverse effect; "retinoid embryopathy" results in many cardiovascular, neurological and limb defects. The risk is high (as for thalidomide), the effect is pharmacological and independent of dosage, and is thought to last up to 1 month after stopping the drug, after which pregnancy is safe. ${ }^{6}$

Mucocutaneous side-effects. Initial worsening of acne; xerosis and cheilitis; retinoid dermatitis; epistaxis; staphylococcal infections of the skin, often of the nail folds, to cause paronychia; pyogenic granulomas of the nail folds are often seen on the toes; hypertrophic scarring and keloids. ${ }^{6}$ 
Ocular complications. Dry eyes that can persist indefinitely; blepharoconjunctivitis; keratitis with corneal ulceration (rare); recent use of isotretinoin is a contraindication to laser refractive eye surgery. Decreased night vision is a common and potentially dangerous side-effect, making driving a vehicle at night hazardous and is the reason why aviation pilots may not use the drug. Night blindness can persist indefinitely. ${ }^{6}$

Laboratory abnormalities. Liver enzyme disturbance; raised serum lipids (especially triglycerides). ${ }^{6}$

Central nervous system abnormalities. Raised intracranial pressure-isotretinoin must not be combined with tetracyclines or vitamin A. Mood disturbance, depression, inability to concentrate and study can occur as uncommon, unpredictable, idiosyncratic events requiring prompt action. The FDA recommends close monitoring of patients treated with isotretinoin for symptoms of depression or suicidal thoughts, irritability, anger, loss of pleasure or interest in sports activities, sleeping too much or too little, changes in weight or appetite, decreased school or work performance, trouble concentrating, mood disturbance, psychosis or aggression. (FDA Alert for Healthcare Professional 07/2005). ${ }^{7}$

Musculoskeletal abnormalities. Myalgia and arthralgia occur more frequently in patients who also engage in heavy exercise, seen in $2-5 \%$ of cases. CK-levels may become markedly raised. Diffuse interosseous skeletal hyperostosis can occur after prolonged use. Premature closure of epiphyses is possible.

Gastro-intestinal side-effects. Some studies found that isotretinoin can cause ulcerative colitis, while others deny it. ${ }^{8}$

\section{Precautions to be taken before initiation of OIT treatment:}

- All patients (male and female) have to sign an information sheet where the side-effects and dangers of the drug are pointed out and where they undertake to comply with contraceptive directives.

- All patients: Liver enzymes (AST, ALT and GGT) and fasting total cholesterol and triglycerides have to be normal.

- Female patients: Pregnancy test has to be negative. Effective contraception has to start immediately.

First tablets are taken on the third day of the next normal menstrual period.

\section{Precautions during treatment OIT:}

- Effective contraception throughout and up to one month after the last tablets were taken.

- Liver enzymes and lipids as above after one month; if normal again, it need not be repeated during the rest of the treatment.

\section{Adjunctive therapies}

Comedo extraction: This is often done to remove macrocomedones, especially after the use of OIT. For other indications, it has little value if not combined with topical retinoids.
Light therapy: Blue light therapy has become popular and undoubtedly has some benefit, but hardly ever as monotherapy. It has not been proven to be equal to or more effective than the conventional drugs outlined above and it is very costly. One important indication for this method is acne in pregnancy, where we cannot use any retinoids, the preferred antibiotics or anti-androgens.

Moisturisers and masks: These have no value and usually worsen the acne; should be avoided.

Vitamin A orally: This can be effective, but in toxic doses; oral isotretinoin is much safer and more effective.

\section{Strategy to follow when a patient fails to respond favourably}

\section{Non-drug related reasons: ${ }^{4}$}

- Severe seborrhoea (oiliness) - consider more potent face wash

- Check (again) exposure of patient to acne-provoking agents/ drugs

- Check stress and diet - consider lower glycaemic diet

- Check comedogenicity of facial make-up and moisturizing cream - avoid altogether

- Macrocomedones - mechanically remove comedones

- Significant P. acnes component - change to retinoid/BPO fixed combination

- P. acnes resistance - change to oral isotretinoin

- Gram-negative folliculitis - change to oral isotretinoin

- Endocrine abnormality (see below) - investigate and address.

Drug-related reasons: ${ }^{4}$

- Females: Check (again) type of contraception - change if necessary, as indicated above

- Retinoid may be inappropriate for skin type or environmental conditions - change retinoid formulation from gel to cream (dry climates) or cream to gel (humid climates)

- Retinoid concentration may be too low - change to higher concentration of retinoid

- Check type and dose of oral antibiotic - correct if needed

- Oral isotretinoin taken on empty stomach - ensure drug is taken with a fatty meal

- Initial flare of acne on isotretinoin - temporarily lower dose and/or add low dose prednisone for two weeks.

\section{Poor adherence: ${ }^{4}$}

- Check if treatment was only applied to spots - patient education to use correctly

- Infrequent application - check frequency and quantity used per month

- Infrequent use of oral drug - check frequency of taking oral treatment

- Adverse events - check and address if necessary

- Patient misunderstands drug effects - educate patient. 


\section{Adverse events:}

- Retinoid irritation - reduce frequency of application to alternative days or change to lower concentration of retinoids or different formulation (change gel to cream)

- Overuse of cleansers - use cleanser with $\mathrm{pH}$ around 5, infrequently

- Possible contact dermatitis and photosensitivity - investigate

- Gastric side-effects of cyclines - take with a meal (will not significantly alter effectivity).

\section{When to investigate for underlying endocrine abnormality}

This is sometimes justified in female patients, especially in cases of severe or recalcitrant acne. It is hardly ever relevant in males. Routine endocrine investigations are not warranted.

Investigations for hyperandrogenism are warranted when the following are present:

- Menstrual abnormalities

- Age of onset after 25 years

- Other signs of polycystic ovary syndrome

- Hirsutism

- Acanthosis nigricans and lipoatrophy

- Virilisation

Baseline investigations should then include:

- Thorough physical, including gynaecological, history and examination

- Blood tests, including levels of total serum testosterone, dehydroepiandrosterone sulphate (DHEAS), LH, FSH, fasting glucose, HBa1c, serum cortisol, serum lipids

- Appropriate imaging for ovarian and adrenal pathology.

Any abnormalities discovered should be managed or referred appropriately.

\section{When to refer to a dermatologist}

Acne cases should preferably be referred in the following situations:

- All cases of acne fulminans (ulcerating acne)

- Nodulocystic acne with scarring, especially acne conglobata

- Underlying endocrine abnormalities

- Uncertain diagnosis

- Severe treatment-related adverse events

- Recalcitrant papulopustular or nodular acne.

\section{Conclusion}

It is hoped that this brief summary and algorithm will present a practical management strategy for acne vulgaris in general practice. If applied, this may contribute to great improvement in the outcomes of acne treatment.

\section{References}

1. Strauss JS, Krowchuk DP, Leyden JJ, et al. Guidelines of care for acne vulgaris management. J Am Acad Dermatol. 2007;56(4):651-63.

2. Thiboutot $D$, Gollnick HP, Bettoli $V$, et al. New insights into the management of acne: An update from the Global Alliance to Improve Outcomes in Acne Group. J Am Acad Dermatol. 2009;60(5):S1-S50.

3. Nast A, Dreno B, Bettoli V, et al. European evidence-based (S3) guidelines for the treatment of acne. J Eur Acad Dermatol Venereol. 2012;26 Suppl 1:1-29.

4. Gollnick H, Bettoli V, Lambert J, et al. A consensus-based practical and daily guide for the treatment of acne patients. J Eur Acad Dermatol Venereol. 2016;30:1480-90.

5. Sinclair W, Jordaan HF. Acne Guidelines 2005 update. S Afr Med J. 2005;95:883-92.

6. Sinclair W. The rational use of systemic isotretinoin: a call for moderation. $\mathrm{S} \mathrm{Afr}$ Med J. 2012; 102(5):282-4.

7. Goodfield MJD, Cox NH, Browser A, et al. Advice on the safe introduction and continued use of isotretinoin in acne in the UK, 2010. Br J Dermatol. 2010;162:1172-9.

8. Shale M, Kaplan GG, Panaccione R, Ghosh R. Isotretinoin and intestinal inflammation: what gastroenterologists need to know. Gut. 2009;58:737-41. 